\title{
Risk and protective factors for drug dependence in two Moroccan high-risk male populations
}

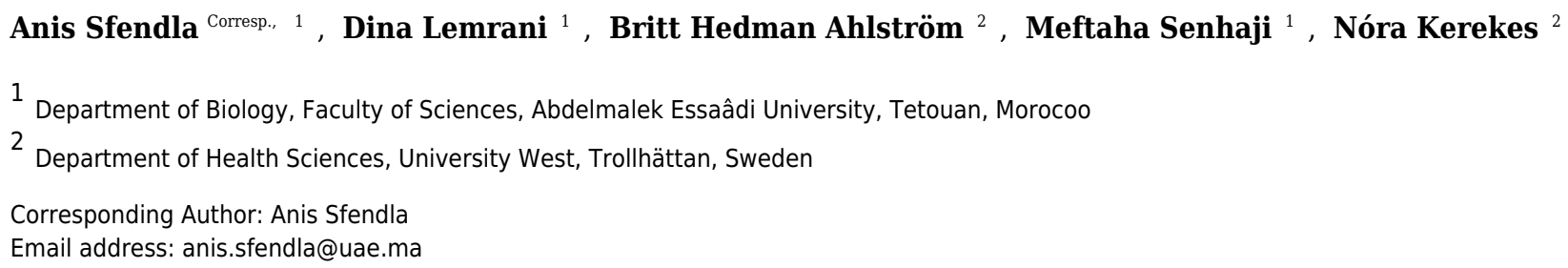

Background. Substance use is linked to biological, environmental, and social factors. This study provides insights on protective and risk factors for drug dependence in two Moroccan, high-risk, male samples. Methods. Data from the "Mental and Somatic Health without borders" (MeSHe) survey were utilized in the present study. The MeSHe survey assesses somatic and mental health parameters by self-report from prison inmates $(n=177)$ and outpatients from an addiction institution $(n=54)$. The 'Drug dependence' and the 'No drug dependence' groups were identified based on the Arabic version of the Drug Use Disorder Identification Test's (DUDIT) validated cutoff for identifying individuals with drug dependence, specifically in Morocco. Results. Majority of participants who had at least high school competence (67.6\%), were living in a partnership (53.7\%), were a parent (43.1\%), and/or had a job (86.8\%) belonged to the 'No dependence' group, while the presence of mental health problems was typical among the 'Drug dependence' group (47.4\%). Multivariable regression model $\left(\chi^{2}(\mathrm{df}=5, N=156)=63.90, p<.001\right)$ revealed that the presence of depression diagnosis remains a significant risk factor, while a higher level of education, having a child, and being employed are protective factors from drug dependence. Discussion. Findings support the importance of increasing academic competence and treating depression as prevention from the persistence of drug addiction in male high-risk populations. 
1 Risk and protective factors for drug dependence in two Moroccan high-risk

2

3

4

5

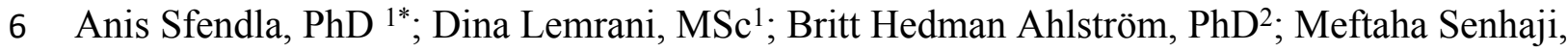

$7 \mathrm{PhD}^{1}$ and Nóra Kerekes, $\mathrm{PhD}^{2}$

8

9

10

11

12

13

14

15

16

17

18

19

20

21

22

23

24

25

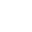

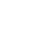

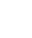

8

${ }^{1}$ Department of Biology, Faculty of Sciences, Abdelmalek Essaâdi University, Tetouan,

Morocco

${ }^{2}$ Department of Health Sciences, University West, Trollhättan, Sweden

* Corresponding author:

Anis Sfendla ${ }^{1}$

17 E-mail: anis.sfendla@gmail.com

Address: Boite Postale 18046 EL WIFAK Meknes, Morocco 
Abstract

29

Background. Substance use is linked to biological, environmental, and social factors. This study provides insights on protective and risk factors for drug dependence in two Moroccan, high-risk, male samples.

Methods. Data from the "Mental and Somatic Health without borders" (MeSHe) survey were utilized in the present study. The MeSHe survey assesses somatic and mental health parameters by self-report from prison inmates $(n=177)$ and outpatients from an addiction institution $(n=54)$. The 'Drug dependence' and the 'No drug dependence' groups were identified based on the Arabic version of the Drug Use Disorder Identification Test's (DUDIT) validated cutoff for identifying individuals with drug dependence, specifically in Morocco.

Results. Majority of participants who had at least high school competence (67.6\%), were living in a partnership (53.7\%), were a parent (43.1\%), and/or had a job (86.8\%) belonged to the 'No dependence' group, while the presence of mental health problems was typical among the 'Drug dependence' group (47.4\%). Multivariable regression model $\left(\chi^{2}(\mathrm{df}=5, N=156)=63.90, p<\right.$ .001) revealed that the presence of depression diagnosis remains a significant risk factor, while a higher level of education, having a child, and being employed are protective factors from drug dependence. Discussion. Findings support the importance of increasing academic competence and treating depression as prevention from the persistence of drug addiction in male high-risk populations. 
48

49

50

51

52

53

54

55

56

57

58

59

60

61

62

\section{Introduction}

The problem of substance use has become a worldwide burden affecting not only individuals' health and well being, but also society's economic development and environmental sustainability (Cartwright, 2008; Chen \& Lin, 2009). In 2014, it was estimated that global drug use prevalence was $5.2 \%$, reflected by 247 million people who had used drugs during the previous year, and while almost 29 million people had substance use disorder, only one in six received treatment (UNODC, 2016).

An increasing trend in drug use has been detected in developing countries, with cannabis as the primary drug of abuse followed by amphetamine-type stimulants, ecstasy (MDMA), and opium derivatives (Odejide, 2006; Pletzer et al., 2011). Generally, the amount of health lost related to drug and alcohol use is higher in low- and middle-income countries than those in high-income countries (Collins et al., 2011). For example, in 2009 drug use disorders in low-income countries accounted for a loss of 6.5 disability-adjusted life years (DALYs), compared with the loss of 1.9 DALYs in high-income countries (Collins et al., 2011). While there are no newer reports presenting actual infomration specifically for developing countries, globally it has been shown that DALYs caused by mental and substance use disorders increased from 7.8 in 2005 to 11 in 2013 (Murray et al., 2015), suggesting that the 6.5 DALYs reported specifically for developing countries for nine years ago, today is even more serious.

Morocco is a unique African Arabic developing country under robust European influences, with a dominant Islamic culture, which itself strongly regulates and punishes drug and alcohol use. However, Morocco is considered one of the main producers of cannabis resin worldwide (INCB, 2005), which makes the concern over drug use and its previously suggested health and societal effects (Kessler et al., 1994;Chen \& Lin, 2009) highly relevant. The latest prevalence of drug use reported in this country was $5.8 \%$ (Kadri et al., 2010), a comparable prevalence with the global 
72 number reflected by 5\% in 2010 (UNODC, 2016).

73 Substance use is linked to multiple negative consequences, including co-occurrence of other

74 psychiatric disorders, somatic health problems, social marginalization, and even criminality

75 (Kessler et al., 1994). A considerable amount of research has affirmed that the negative

76 outcomes of many psychiatric disorders (such as anxiety, depression, psychosis, and

77 schizophrenia) are related to co-existing substance use, while abstainers may have more

78 favorable outcomes (such as better health status, life satisfaction, and education achievements)

79 (Brook et al., 2002, 2011; Lennings, Copeland \& Howard, 2003; Ellickson, Martino \& Collins,

80 2004; Fazel et al., 2009; Van Ryzin, Fosco \& Dishion, 2012). It is also well documented that the

81 prevalence of substance use is high in samples of prison inmates (Fazel, Bains \& Doll, 2006),

82 and those living in bad social circumstances (Spooner \& Hetherington, 2004).

83 Regarding interpersonal risk and protective factors, longitudinal studies have found that anxiety

84 and depression are linked to the subsequent development of substance use, with nearly one-third

85 of major depressive patients having a substance use disorder (Davis et al., 2008). Moreover, it

86 was found that being single and the absence of employment were the strongest societal risk

87 factors (Lennings, Copeland \& Howard, 2003; Davis et al., 2008; Brook et al., 2011; Vivek et

88 al., 2011; Stone et al., 2012).

89 It is known that lower socioeconomic status of developing countries is associated with often

90 insufficient detection (both in quantity and quality) of somatic and psychiatric problems and with

91 the lack of therapy and rehabilitation possibilities (Patel, 2007). From a research standpoint,

92 epidemiological studies on psychiatric disorders are quite rare in developing countries (Dewing

93 et al., 2006; Plüddemann, Myers \& Parry, 2008). It is even more difficult to find scientific works

94 focusing on risk and protective factors for the development of addiction in Moroccan samples. 
95 To our knowledge, only one study has investigated the associated factors of psychoactive

96 substance use in Morocco. This study collected data from middle- and high-school students and

97 revealed that male gender, secondary school level (13-16 years of age), smoking tobacco, living

98 with family members who use tobacco, and feeling insecure within family environment were the

99 risk factors predicting drug use (Zarrouq et al., 2016).

100 The scarcity of research indirectly limits the development of effective preventive and adequate

101 rehabilitation and treatment programs. With the present work we aim to contribute to this

102 research area, investigating protective and risk factors for drug dependence in two, Moroccan

103 samples: male prison inmates and male psychiatric outpatients with a substance use disorder

104 diagnosis.

\section{Participants \& Methods}

\section{Study design}

107 The 'Mental and Somatic Health without borders' (MeSHe) international project

108 (http://meshe.se) aims to identify culture specific personality-, behavioral-, physical-, and

109 psychiatric symptoms to improve upon possibilities for an early identification of those who may

110 require (social and/or psychological) help with their substance use. The project assesses data

111 from different study populations with the help of a standardized survey (the MeSHe survey),

112 which includes, besides other validated inventories, the self-reported Drug Use Identification

113 Disorder Test (DUDIT) (Berman et al., 2005). Within the frame of this international project, data

114 from two high-risk male samples were collected in Morocco, during the period between June

1152013 and July 2014. General Directors of the Prison Administration and Rehabilitation and the

116 Hasnouna Association Drug Users Support center consented to the study.

\section{Participants}


118 There were too few female participants in the clinical sample and no data collected from female

119 inmates therefore data from male outpatients with substance use disorder (SUD) diagnosis

$120(n=54)$ and male prison inmates $(n=177)$ were utilized. Inmates were recruited from local

121 “Toulal 2" prison in Meknes, Morocco; while outpatients were recruited from a medical and

122 psychological prevention center in Tangier, Morocco. The prison inmate sample reflects $7.2 \%$ of

123 those incarcerated during assessment; whereas, all of the male outpatients chose to participate

124 (100\%). For further clarifications about clinical and inmate sample, survey administration and

125 study design please refer to Sfendla and colleagues publication (2017).

126 Measures

127 The Arabic version of DUDIT was recently validated (Sfendla et al., 2017). The DUDIT consists

128 of 11 simple questions, which assess the frequency of drug use during the past 12 months via

129 self-reports. For the identification of those with drug dependence (the 'Drug dependence' group),

130 the previously validated cutoff score of 3.0 was used (Sfendla et al., 2017); therefore, those

131 scoring in the DUDIT zero, one, or two points formed the group named as the "No drug

132 dependence'.

133 The MeSHe survey includes socio-demographic variables (education, employment, partnership,

134 and parenthood) in its background questionnaire section. Education levels were categorized into

135 groups of "high education" (that is, those who achieved high school and/or college/university

136 education) and "low education" (participants reporting no education or only

137 elementary/secondary school education). Employment was recorded based on a binary reply

138 where current job was indicated (Yes/No). For inmates, the answer should have reflected the

139 state prior to incarceration. Partnership status was categorized into "living in a partnership" if the

140 participant indicated that he was married or living with a partner; and "single" when the 
141 participant indicated that he was divorced, separated, or living alone. Finally, parenthood was

142 also recorded into a binary variable based on having or not having children.

143 The MeSHe survey also assesses information about the existence of previously received (from a

144 physician) psychiatric diagnosis of any of the following: depression, anxiety disorder, obsessive

145 compulsive disorder (OCD), post-traumatic stress disorder (PTSD), bipolar disorder, other

146 personality disorders, eating disorder, or schizophrenia.

\section{Statistical Analysis}

148 When comparing descriptors between the groups of 'Drug dependence' and 'No drug

149 dependence', Student $t$-test was used for continuous variables (age and drop-out education age),

150 while Chi-square test $\left(\chi^{2}\right)$ was used for categorical variables (education, profession, marital

151 status, children, and psychiatric disorders), where the degree of the association was assessed by

152 the phi coefficient $(\phi)$. Cohen's criteria were applied to $p h i$ coefficient where .10 indicated a

153 small effect, .30 a medium effect, and .50 a large effect (Cohen, 1988). When the Chi-square

154 assumption was violated, likelihood ratios were used. Risk Ratios (RR) were reported reflecting

155 the probability of different psychiatric disorders in the 'Drug dependence' group divided by the

156 probability of psychiatric disorders in the 'No drug dependence' group. Only significant

157 descriptors were placed in the multivariable prediction model. The multivariable logistic

158 regression model was used to measure their association to drug dependence. The dependent

159 variable was coded 0 for those belonging to the 'No drug dependence' group and 1 for those

160 belonging to the 'Drug dependence' group. Thereby, a negative beta coefficient would indicate

161 that an increase in the continuous variable or a "yes" answer for the categorical variables are

162 associated with a decrease in the probability of a score of 1 in the independent variable (being

163 drug dependent). Consequently, a positive beta coefficient suggests an increased probability of 
164 being drug dependent with increased continuous variable or with the presence (yes) of any

165 categorical variable. All statistical analyses were conducted by SPSS version 21 for windows

166 (IBM), with significance level at $5 \%$.

\section{Ethical Considerations}

168 The study process was in agreement with the Helsinki declaration (World Medical Association,

169 2008). Participation was voluntary and anonymous. The cover page of the survey informed the

170 participants about the questionnaire's content and the aims of the study and they also received

171 oral information, after which they could decide whether they want to answer the survey. All

172 participants were assured that their answers would not affect sentences (in case of inmates) or

173 their treatment plans (for those who were outpatients). Completion of the survey was considered

174 as consent of participation. The use of the survey was approved by the Directorate-General of

175 Prison Administration and Rehabilitation (for inmates) (case identifier number: 13993), and by

176 the Director of the Medical and Psychological Prevention Center (for those who were

177 outpatients).

\section{Results}

179 The clinical outpatients sample mean age was $38.3(\mathrm{SD}=8.3)$, and the mean age of the prison 180 inmate sample was $30.8(\mathrm{SD}=10.6)$. No significant differences were detected in age $(p=.73)$, the 181 age when participants finished education $(p=.65)$, and the prevalence of selected psychiatric

182 problems between these two samples $(p=.25)$.

\section{Comparison between 'Drug dependence' and 'No drug dependence' groups}

184 Significant differences were found when separating participant with drug dependence from those 185 without drug dependence (Table 1). Fewer participants in the 'Drug dependence' group lived in a 186 partnership, had a child, had a high level of education, or were employed, compared to the 'No 
187 drug dependence' group ( $p<.001$, in each case). In detail, over two-thirds (67\%) of those

188 indicating active drug use and drug dependence during the past 12 months had a low education

189 level, compared to only one-third (32\%) of those who reported no or minimal (less than 3 points

190 in DUDIT) drug use. The Chi-square test for independence indicated a significant difference,

191 with a medium effect size, in the level of education $\left(\chi^{2}(1, \mathrm{n}=189)=21.3, p<.001, p h i=.34\right)$

192 between the two groups. Most (82\%) participants from the 'Drug dependence' group and more 193 than half (57\%) from the 'No drug dependence' group reported being single, and in a similar 194 ratio they reported not having children ( $80 \%$ and $46 \%$, respectively). Chi-square tests for 195 independence indicated a weak but significant difference in marital status $\left(\chi^{2}(1, n=190)=13.2\right.$, 196 $p<.001, p h i=.26)$ and in parenthood status $\left(\chi^{2}(1, \mathrm{n}=184)=21.4, p<.001, p h i=.34\right)$ between

197 those with drug dependence and those who were not active or no users. Similarly, the unemployment rate was more than three times higher in the 'Drug dependence' group (43\%) than in the 'No drug dependence' group (13\%). The Chi-square test showed a significant and medium-strong association between active drug dependence and unemployment $\left(\chi^{2}(1, n=187)=\right.$ $17.4, p<.001, p h i=.30)$. While the presence of any psychiatric disorder was not significantly different between the groups (47\% and $38 \%$, respectively), a comparison between the prevalence of defined disorders revealed that depression was a significantly more frequent coexisting problem in those with drug dependence (22\%) compared to those with no dependence or no use $(3 \%)\left(\chi^{2}(1, \mathrm{n}=167)=9.96,(p<.001), p h i=.24\right)$. - 7.654) higher risk of depression compared to the 'No drug dependence' group. Likewise, the risk of having obsessive-compulsive disorder (OCD) was 2.7 times (95 \% CI: 2.699 - 3.434) 
210 having a coexisting eating disorder was 25\% lower in the 'Drug dependence' group compared to

211 the 'No drug dependence' group.

212

213 Multivariable prediction model

214 Only those variables that significantly differed between the groups were fitted in the

215 multivariable prediction model. The final model included the following factors: education level,

216 having a child, being employed, being in a partnership, and existing diagnosis of depression.

217 Table 2 presents the multivariable associations of sociodemographic and clinical correlates with

218 active drug dependence. The model containing all predictors was statistically significant $\left(\chi^{2}(\mathrm{df}=\right.$

$2195, N=156)=63.90, p<.001)$, showing that the model could explain over $60 \%$ of drug

220 dependence. Four of the predictor variables (education level, existence of employment, having

221 any children, and diagnosis of depression) had a highly significant contribution to the model. The

222 strongest predictor of drug dependence in this model was the existence of a diagnosis of

223 depression. Participants who had reported the existence of a diagnosis of depression were 17

224 times more likely to be also identified by the DUDIT as people with drug dependence. On the

225 other hand, high education level, having a child, and being employed were significant protective

226 factors from being active in drug use and dependence. Participants with a higher education

227 profile were about seven times less likely to have a drug dependence. Having children and being

228 employed also decreased the likelihood of being a drug dependent person by about four times

229 and five-and-a-half times, respectively.

230 Discussion

231 This study contributes to the rarely studied field of drug addiction in Morocco and provides

232 insights into protective and risk factors utilizing data from Moroccan samples of prison inmates 
233 and individuals with a previous diagnosis of drug use disorder, both samples with a high risk of

234 active drug use and dependence. In the Moroccan high-risk populations' education level,

235 employment, partnership, parenthood, and the diagnosis of depression were significantly

236 associated with being actively drug dependent. Specifically, the study showed depression is the

237 factor that has the strongest association with drug dependence, while higher level of education,

238 being employed, and having a child can be significant protective factors against it.

239 Considering socio-demographic factors, previous studies have also indicated that high level of

240 education is a protective factor from substance use (Fitzpatrick, Piko \& Wright, 2005; Mirza \&

241 Mirza, 2008; Piko \& Kovács, 2010). While these studies assessed protective factors among

242 younger samples and in other nations, results in the present confirm the same findings in high-

243 risk Moroccan populations, specifically indicating that education is the strongest protective

244 factor even in the samples of clinical and incarcerated individuals. Another significant protective

245 factor in high-risk samples was employment. Previous empirical evidence has persistently

246 reported that unemployment is often coupled with substance use (Henkel, 2011; Compton et al.,

247 2014; Lee et al., 2015). In addition, international research has previously shown a negative

248 association between drug use and employment (French, Roebuck \& Alexandre, 2001; DeSimone,

249 2002). The association between unemployment and drug use has been suggested to be mediated

250 by personality traits - such as stress reactivity or impulsivity - that could relate unemployment to

251 substance use (Compton et al., 2014). Assuming that the prison inmate sample may consist of

252 individuals with a high level of impulsivity (Cuomo et al., 2008; Bernstein et al., 2015), then the

253 previously suggested interaction could also be tested in this study; however no direct information

254 was available about the level of impulsivity or impulse control in the Moroccan samples.

255 The results support previous statistics, showing that the proportion of adults reporting active use 
256 of illegal drugs is increased with unemployment (Badel \& Greaney, 2013).

257 Of the other socio-demographic factors, while relationship was not a significantly affecting

258 factor of active drug dependence in the Moroccan high-risk populations, parenthood emerged as

259 an important protective factor. This is in accordance with previous findings showing that

260 parenthood is most often linked to limited or no drug use, and therefore custodial parenthood

261 results in the decline of the risk of drug use (Bachman et al., 2002; Fergusson, Boden \&

262 Horwood, 2012). It is important to mention that age by itself was not correlated to substance use;

263 therefore, the protective action of parenthood and employment is not mediated by older age.

264 As regard to the existence of (any) psychiatric problem, no association was found with drug

265 dependence. However, the presence of depression significantly increased the risk of coexisting

266 drug dependence in high-risk samples. This result is in accordance with previous findings

267 showing that drug use and depression coexist in clinical and prison inmate samples (Ross, 1988;

268 Rowe et al., 1995; Vreugdenhil et al., 2003; Najt, Fusar-Poli \& Brambilla, 2011). While many

269 studies have confirmed the association between depression and drug use, the explanation of their

270 link is still not complete. Bovasso (2001) found that adult participants with an initial diagnosis of

271 cannabis use are four times more likely to have depressive symptoms at a follow-up assessment

272 than those with no diagnosis of cannabis use. Other longitudinal studies have also suggested that

273 substance use precedes depressive symptoms even in adults (Weller \& Halikas, 1985; Grant,

274 1995; Angst, 1996).

275 The findings of the present study are consistent with the results of previous studies on risk and

276 protective factors, and also of high relevance to substance use treatment and prevention programs

277 targeting prison inmates and psychiatric patients. Outpatient centers should help individuals to

278 find social support and job opportunities, improve their educational level, and provide adequate 
279 medical care even for their depressive symptoms, which is possible when utilizing integrative

280 treatments (Weisner et al., 2001). Also, our work by showing that social factors are indeed

281 protective factors for drug dependence even in high risk groups, supports the newly launched

282 theories questioning the dominant influence of the biological, "brain disease model of addiction"

283 while suppressing the psychosocial perspectives of addiction (Heather et al., 2018).

284 While the present study is highly important due to the scarcity of psychiatric data collection and 285 studies in developing countries, it also has several limitations. The DUDIT only refers to the 286 pattern of use and did not make possible specific identification of the type of drug use. Not

287 knowing which substance or substances the participants in this study were using may result in confounding with respect to which psychiatric conditions were of most concern. This should be considered in the general recommendations for community treatment services. The relatively small sample size within each subpopulation could be argued to cause possible type II errors in our analyses, and definitely limit the generalizability of the results, mainly considering prison inmates as only $7.2 \%$ of the total incarcerated population participated in the study. Importantly, the study sample included only male participants, therefore the findings and recommendations may not be relevant to the female population. Moreover, the cross-sectional design of the MeSHe study limits the possibility of causality analyses. Finally, the existence of psychiatric diagnoses was self-reported and not gathered from inpatient registers, but the study design (anonymous participation) does not allow any follow-up or merging of the collected data. Based on these limitations it could be recommended that a larger study be designed, which addresses the potential selection bias raised above, and which incorporates measures that will make separate analyses possible for different types of drugs. Further research investigating the 
301 effectiveness of treatment for co-occurring substance use and psychiatric disorders in a low- and

302 middle-income country setting, such as Morocco, is also warranted.

\section{Conclusion}

304 The results of the present study emphasize the need for adequate psycho-educational programs

305

306

307

308

309

310

311

312

313

314

315

316

317

318

319

320

321

322

323

324

325

326

327

328

329 alongside the established medical treatments and social support for individuals with drug dependence. Our study shows that integrated treatment is required for addiction, where not only one medical problem is addressed (substance use disorder), but also comorbid psychiatric problems (such as depression); importantly, these should always be completed with education and social-psychiatric care. In Morocco, medical and psychological prevention centers are linked to associative work mainly under the frame of risk reduction, psychosocial support, and employment integration for drug users. There is a need to increase the number of these outpatient care centers with qualified personal in psychiatry, also in Morocco, to be able to decrease the prevalence of drug use and dependence, consequently reducing the prevalence of negative results (such as criminality, chronic mental illness, self-harm behavior, and even early death).

\section{References}

Angst J. 1996. Comorbidity of mood disorders: A longitudinal prospective study. British Journal of Psychiatry 168:31-37.

Bachman JG., O’Malley PM., Schulenberg JE., Johnston LD., Bryant AL., Merline AC. 2002. The Decline of Substance Use in Young Adulthood: Changes in Social Activities, Roles, and Beliefs. RMA. DOI: 10.1017/CBO9781107415324.004.

Badel BA., Greaney B. 2013. Exploring the Link between Drug Use and Job Status in the U.S. The Regional Economist 21:1-3.

Berman AH., Bergman H., Palmstierna T., Schlyter F. 2005. Evaluation of the Drug Use Disorders Identification Test (DUDIT) in criminal justice and detoxification settings and in a Swedish population sample. European Addiction Research 11:22-31. DOI: $10.1159 / 000081413$.

Bernstein MH., McSheffrey SN., van den Berg JJ., Vela JE., Stein LAR., Roberts MB., Martin RA., Clarke JG. 2015. The association between impulsivity and alcohol/drug use among prison inmates. Addictive Behaviors 42:140-143. DOI: 10.1016/j.addbeh.2014.11.016. 
330

331

332

333

334

335

336

337

338

339

340

341

342

343

344

345

346

347

348

349

350

351

352

353

354

355

356

357

358

359

360

361

362

363

364

365

366

367

Bovasso GB. 2001. Cannabis abuse as a risk factor for depressive symptoms. The American journal of psychiatry 158:2033-2037. DOI: 10.1176/appi.ajp.158.12.2033.

Brook DW., Brook JS., Zhang CS., Cohen P., Whiteman M. 2002. Drug use and the risk of major depressive disorder, alcohol dependence, and substance use disorders. Archives of General Psychiatry 59:1039-1044. DOI: 10.1001/archpsyc.59.11.1039.

Brook JS., Lee JY., Finch SJ., Koppel J., Brook DW. 2011. Psychosocial Factors Related to Cannabis Use Disorders. Substance Abuse 32:242-251. DOI: 10.1080/08897077.2011.605696.

Cartwright WS. 2008. Economic costs of drug abuse: Financial, cost of illness, and services. Journal of Substance Abuse Treatment 34:224-233. DOI: 10.1016/j.jsat.2007.04.003.

Chen C-Y., Lin K-M. 2009. Health consequences of illegal drug use. Current opinion in psychiatry 22:287-92.

Cohen J. 1988. Statistical Power Analysis for the Behavioral Sciences.

Collins PY., Patel V., Joestl SS., March D., Insel TR., Daar AS. 2011. Grand challenges in global mental health: A consortium of researchers, advocates and clinicians announces here research priorities for improving the lives of people with mental illness around the world, and calls for urgent action and investment. Nature 475:27-30. DOI: 10.1038/475027a.

Compton WM., Gfroerer J., Conway KP., Finger MS. 2014. Unemployment and substance outcomes in the United States 2002-2010. Drug and Alcohol Dependence 142:350-353. DOI: 10.1016/j.drugalcdep.2014.06.012.

Consortium TWHOWMHS. 2004. Prevalence, severity, and unmet need for treatment of mental disorders in the world health organization world mental health surveys. JAMA 291:25812590 .

Cuomo C., Sarchiapone M., Giannantonio M Di., Mancini M., Roy A. 2008. Aggression, impulsivity, personality traits, and childhood trauma of prisoners with substance abuse and addiction. American Journal of Drug and Alcohol Abuse 34:339-345. DOI: 10.1080/00952990802010884.

Davis L., Uezato A., Newell JM., Frazier E. 2008. Major depression and comorbid substance use disorders. Current Opinion in Psychiatry 21:14-18. DOI: 10.1097/YCO.0b013e3282f32408.

DeSimone J. 2002. Illegal Drug Use and Employment. Journal of Labor Economics 20:952-977. DOI: $10.1086 / 342893$.

Dewing S., Plüddemann A., Myers BJ., Parry CDH. 2006. Review of injection drug use in six African countries: Egypt, Kenya, Mauritius, Nigeria, South Africa and Tanzania. Drugs: Education, Prevention and Policy 13:121-137. DOI: 10.1080/09687630500480228.

Ellickson PL., Martino SC., Collins RL. 2004. Marijuana use from adolescence to young adulthood: Multiple developmental trajectories and their associated outcomes. Health Psychology 23:299-307. DOI: 10.1037/0278-6133.23.3.299. 
402

403

404 405

Faupel CE. 1988. Heroin Use, Crime and Employment Status. Journal of Drug Issues 18:467479. DOI: $10.1177 / 002204268801800311$.

Fazel S., Bains P., Doll H. 2006. Substance abuse and dependence in prisoners: A systematic review. Addiction 101:181-191. DOI: 10.1111/j.1360-0443.2006.01316.x.

Fazel S., Långström N., Hjern A., Grann M., Lichtenstein P. 2009. Schizophrenia, Substance Abuse, and Violent Crime. JAMA 301:2016-2023. DOI: 10.1001/jama.2009.675.

Fergusson DM., Boden JM., John Horwood L. 2012. Transition to parenthood and substance use disorders: Findings from a 30-year longitudinal study. Drug and Alcohol Dependence 125:295-300. DOI: 10.1016/j.drugalcdep.2012.03.003.

Fitzpatrick KM., Piko BF., Wright DR. 2005. A Tale of Two Cities: Health-Compromising Behaviors Between Hungarian and American Youth. Sociological Studies of Children and Youth 10:189-212. DOI: 10.1016/S1537-4661(04)10010-X.

French MT., Roebuck MC., Alexandre PK. 2001. Illicit Drug Use, Employment, and Labor Force Participation. Southern Economic Journal 68:349-368. DOI: 10.2307/1061598.

Grant BF. 1995. Comorbidity between DSM-IV drug use disorders and major depression: results of a national survey of adults. Journal of Substance Abuse 7:481-497. DOI: 10.1016/08993289(95)90017-9.

Henkel D. 2011. Unemployment and substance use: a review of the literature (1990-2010). Curr Drug Abuse Rev 4:4-27. DOI: 10.2174/1874473711104010004.

Heather N., Best D., Kawalek A., Field M., Lewis M., Rotgers F., Wiers RW., Heim D. 2018. Challenging the brain disease model of addiction: European launch of the addiction theory network. Addiction Research and Theory 26:249-255. DOI: 10.1080/16066359.2017.1399659.

INCB. 2005. Report of the International Narcotics Control Board 2004.

Kadri N., Agoub M., Assouab F., Tazi MA., Didouh A., Stewart R., Moussaoui D. 2010. Moroccan national study on prevalence of mental disorders: A community-based epidemiological study. Acta Psychiatrica Scandinavica 121:71-74. DOI: 10.1111/j.16000447.2009.01431.x.

Kessler RC., McGonagle KA., Zhao S., Nelson CB., Hughes M., Eshleman S., Wittchen HU., Kendler KS. 1994. Lifetime and 12-month prevalence of DSM-III-R psychiatric disorders in the United States. Results from the National Comorbidity Survey. Archives of general psychiatry 51:8-19. DOI: 10.1001/archpsyc.1994.03950010008002.

Lee JO., Hill KG., Hartigan LA., Boden JM., Guttmannova K., Kosterman R., Bailey JA., Catalano RF. 2015. Unemployment and substance use problems among young adults: Does childhood low socioeconomic status exacerbate the effect? Social Science and Medicine 143:36-44. DOI: 10.1016/j.socscimed.2015.08.016.

Lennings CJ., Copeland J., Howard J. 2003. Substance Use Patterns of Young Offenders and Violent Crime. Aggressive Behavior 29:414-422. DOI: 10.1002/ab.10048. 
406

407

408

409

410

411

412

413

414

415

416

417

418

419

420

421

422

423

424

425

426

427

428

429

430

431

432

433

434

435

436

437

438

439

440

441

442

443

Merikangas KR., McClair VL. 2012. Epidemiology of Substance Use Disorders. Human genetics 131:779-789. DOI: 10.1007/s00439-012-1168-0.

Mirza KAH., Mirza S. 2008. Adolescent substance misuse. Psychiatry 7:357-362. DOI: 10.1016/j.mppsy.2008.05.011.

Murray CJL., Barber RM., Foreman KJ., Ozgoren AA., Abd-Allah F. 2015. Global, regional, and national disability-adjusted life years (DALYs) for 306 diseases and injuries and healthy life expectancy (HALE) for 188 countries, 1990-2013: Quantifying the epidemiological transition. The Lancet 386:2145-2191. DOI: 10.1016/S01406736(15)61340-X.

Najt P., Fusar-Poli P., Brambilla P. 2011. Co-occurring mental and substance abuse disorders: A review on the potential predictors and clinical outcomes. Psychiatry Research 186:159-164. DOI: $10.1016 /$ j.psychres.2010.07.042.

Odejide AO. 2006. Status of drug use/abuse in Africa: A review. International Journal of Mental Health and Addiction 4:87-102. DOI: 10.1007/s11469-006-9015-y.

Patel V. 2007. Mental health in low- and middle-income countries. British Medical Bulletin 8182:81-96. DOI: 10.1093/bmb/ldm010.

Piko BF., Kovács E. 2010. Do parents and school matter? Protective factors for adolescent substance use. Addictive Behaviors 35:53-56. DOI: 10.1016/j.addbeh.2009.08.004.

Pletzer K., Ramlagan S., Johnson B., Phaswana-Mafuya N. 2011. Illicit drug use and treatment in South Africa: a review. Substance Use \& Misuse, 2011, Vol.45(13), p.2221-2243 45:122. DOI: 10.3109/10826084.2010.481594.Illicit.

Plüddemann A., Myers BJ., Parry CDH. 2008. Surge in treatment admissions related to methamphetamine use in Cape Town, South Africa: Implications for public health. Drug and Alcohol Review 27:185-189. DOI: 10.1080/09595230701829363.

Ross HE. 1988. The Prevalence of Psychiatric Disorders in Patients With Alcohol and Other Drug Problems. Archives of General Psychiatry 45:1023. DOI: 10.1001/archpsyc.1988.01800350057008.

Rowe MG., Fleming MF., Barry KL., Manwell LB., Kropp S. 1995. Correlates of depression in primary care. The Journal of family practice 41:551-558.

Sfendla A., Zouini B., Lemrani D., Berman AH., Senhaji M., Kerekes N. 2017. Psychometric Properties of the Arabic Version of the Drug Use Disorders Identification Test (DUDIT) in Clinical, Prison Inmate, and Student Samples. International Journal of Behavioral Medicine:1-8. DOI: 10.1007/s12529-016-9623-2.

Spooner C., Hetherington K. 2004. Social determinants of drug use / Catherine Spooner and Kate Hetherington. National Drug and Alcohol Research Centre Sydney:xv,291p.

Stone AL., Becker LG., Huber AM., Catalano RF. 2012. Review of risk and protective factors of substance use and problem use in emerging adulthood. Addictive Behaviors 37:747-775. DOI: 10.1016/j.addbeh.2012.02.014. 
444

445

446

447

448

449

450

451

452

453

454

455

456

457

458

459

460

461

462

463

464

465

466

UNODC. 2016. World drug report. DOI: 10.1007/s13398-014-0173-7.2.

Van Ryzin MJ., Fosco GM., Dishion TJ. 2012. Family and peer predictors of substance use from early adolescence to early adulthood: An 11-year prospective analysis. Addictive Behaviors 37:1314-1324. DOI: 10.1016/j.addbeh.2012.06.020.

Vivek K., Dalal P., Trivedi J., Pankaj K. 2011. Comparision of Sociodemographic Variables in Alcohol and Opioid Dependence. 14.

Vreugdenhil C., Van Den Brink W., Wouters LFJM., Doreleijers T a H. 2003. Substance use, substance use disorders, and comorbidity patterns in a representative sample of incarcerated male Dutch adolescents. The Journal of nervous and mental disease 191:372-8. DOI: 10.1097/01.NMD.0000071585.09816.F4.

Weisner C., Mertens J., Parthasarathy S., Moore C., Lu Y. 2001. Integrating primary medical care with addiction treatment: a randomized controlled trial. Jama 286:1715-23. DOI: joc10050 [pii].

Weller RA., Halikas JA. 1985. Marijuana use and psychiatric illness: a follow-up study. The American journal of psychiatry 142:848-850. DOI: 10.1176/ajp.142.7.848.

World Medical Association. 2008. WMA Declaration of Helsinki- ethical principles for medical research involving human subjects. http://www.wma.net/en/30publications/10policies/b3/.

Zarrouq B., Bendaou B., El Asri A., Achour S., Rammouz I., Aalouane R., Lyoussi B., Khelafa S., Bout A., Berhili N., Hlal H., Najdi A., Nejjari C., El Rhazi K. 2016. Psychoactive substances use and associated factors among middle and high school students in the North Center of Morocco: a cross-sectional questionnaire survey. BMC Public Health 16:1-9. DOI: $10.1186 / \mathrm{s} 12889-016-3143-5$. 


\section{Table $\mathbf{1}$ (on next page)}

Socio-demographic and clinical background of participants 


\section{Table 1:}

2 Socio-demographic and clinical background of participants $(N=191)$

\begin{tabular}{|c|c|c|c|c|c|c|c|}
\hline Background variables & $\begin{array}{c}\text { Total sample } \\
\text { h }(n)\end{array}$ & $\begin{array}{c}\text { Drug } \\
\text { dependence } \\
\text { group }^{\mathrm{f}} \\
(n=119) \\
\end{array}$ & $\begin{array}{l}\text { No drug } \\
\text { dependence } \\
\text { group }^{\mathrm{f}} \\
(n=72) \\
\end{array}$ & $\mathrm{RR}^{\mathrm{g}}$ & $t / \chi^{2}$ & $p$-value & $\phi$ \\
\hline Age M $(s d)$ & $32.7(10.62)$ & $32.52(10.95)$ & $34.01(10.08)$ & & .935 & 0.73 & \\
\hline Education dropout Age & $16.95(7.7)$ & $15.93(4.29)$ & $19.45(3.61)$ & & 4.21 & 0.65 & \\
\hline \multicolumn{8}{|l|}{ Education a $\%(n)$} \\
\hline High educational level & $46 \%(87)$ & $33.1 \%(39)$ & $67.6 \%(48)$ & & \multirow{2}{*}{21.31} & \multirow{2}{*}{$<0.001$} & \multirow{2}{*}{.34} \\
\hline Low educational level & $54 \%(102)$ & $66.9 \%(79)$ & $32.4 \%(23)$ & & & & \\
\hline \multicolumn{8}{|l|}{ Marital Status ${ }^{b}$} \\
\hline In a relationship & $27.9 \%(53)$ & $18.6 \%(22)$ & $43.1 \%(41)$ & & \multirow{2}{*}{13.25} & \multirow{2}{*}{$<0.001$} & \multirow{2}{*}{.26} \\
\hline Single & $72.1 \%(137)$ & $81.8 \%(96)$ & $56.9 \%(31)$ & & & & \\
\hline \multicolumn{8}{|l|}{ Parenthood } \\
\hline Yes & $32.6 \%(60)$ & $20.5 \%(24)$ & $53.7 \%(36)$ & & \multirow{2}{*}{21.4} & \multirow{2}{*}{$<0.001$} & \multirow{2}{*}{.34} \\
\hline No & $67.4 \%(124)$ & $79.5 \%(93)$ & $46.3 \%(31)$ & & & & \\
\hline \multicolumn{8}{|l|}{ Employment } \\
\hline Yes & $67.9 \%(127)$ & $57.1 \%(68)$ & $86.8 \%(59)$ & & \multirow{2}{*}{17.42} & \multirow{2}{*}{$<0.001$} & \multirow{2}{*}{.30} \\
\hline No & $32.1 \%(60)$ & $42.9 \%(51)$ & $13.2 \%(9)$ & & & & \\
\hline \multicolumn{8}{|l|}{ Psychiatric disorders ${ }^{\mathrm{c}}$} \\
\hline Yes $^{c}$ & $44.1 \%(79)$ & $47.4 \%(54)$ & $38.5 \%(25)$ & & \multirow{2}{*}{1.33} & \multirow{2}{*}{0.25} & \\
\hline $\mathrm{No}^{c}$ & $55.9 \%(100)$ & $52.6 \%(60)$ & $61.5 \%(40)$ & & & & \\
\hline Depression & $15 \%(25)$ & $21.5 \%(23)$ & $3.3 \%(2)$ & 6.44 & 9.96 & 0.002 & .24 \\
\hline Anxiety & $20.9 \%(37)$ & $21.2 \%(24)$ & $20.3 \%(13)$ & 1.04 & .02 & 0.88 & \\
\hline $\mathrm{OCD}^{\mathrm{d}}$ & $10.6 \%(18)$ & $13.6 \%(15)$ & $5 \%(3)$ & 2.72 & 3.06 & 0.08 & \\
\hline PTSD $^{\mathrm{e}}$ & $19 \%(34)$ & $19.1 \%(21)$ & $18.8 \%(13)$ & 1.01 & .00 & 0.97 & \\
\hline Bipolar disorder & $3.4 \%(6)$ & $3.8 \%(4)$ & $3.3 \%(2)$ & 1.17 & $.036^{\mathrm{Lh}}$ & 0.85 & \\
\hline Eating disorder & $21.1 \%(38)$ & $18.8 \%(21)$ & $25 \%(17)$ & 0.75 & .992 & 0.32 & \\
\hline Schizophrenia & $5.7 \%(10)$ & $7.3 \%(8)$ & $3.1 \%(2)$ & 2.36 & $1.46^{\mathrm{Lh}}$ & 0.23 & \\
\hline Personality disorder & $9.4 \%(16)$ & $11.1 \%(12)$ & $6.3 \%(4)$ & 1.75 & 1.06 & 0.30 & \\
\hline
\end{tabular}

3

$4{ }^{a}$ Education was divided into high educational level, including those with high school and /or

5 university/college education, and low educational level, including those with no education or

6 only elementary/secondary school education.

$7 \quad \mathrm{~b}$ Living in partnership includes the situations of being married, remarried, and living with a

8 partner. Not living in a partnership comprises being single, divorced, and separated. 
$9 \quad{ }^{\mathrm{c}}$ The existence of Substance Use Disorder diagnoses was excluded from psychiatric diagnoses.

$10{ }^{\mathrm{d}}$ Obsessive Compulsive Disorder.

$11{ }^{\text {e }}$ Post-Traumatic Stress Disorder.

$12{ }^{\mathrm{f}}$ Drug dependence was classified based on DUDIT total score using cutoff score (Cutoff $>=3$

13 drug dependence) and (Cutoff $<3$ no drug dependence).

14 g Risk Ratios reflecting the probability of different psychiatric disorders in the Dependent group

15 divided by the probability of psychiatric disorders in the Non-dependent group.

$16{ }^{\mathrm{h}}$ Listwise deletion was used to handle missing values resulting in different total sample for each 17 analysis and consequently different percentages.

18 Lh Likehood ratio test was used based on the violation of Chi-square assumption. 


\section{Table 2 (on next page)}

Multivariable model for prediction of drug dependence

Table of the multivariable logistic regression model that was used to measure association to drug dependence. 
1 Table 2:

2 Multivariable model for prediction of drug dependence

\begin{tabular}{lcccc}
\hline Predictors & $\beta$ & $p$ & $e^{B} / \mathrm{OR}$ & $95 \% \mathrm{CI}$ \\
\hline Education & -1.862 & $<.001$ & .15 & $.065-.374$ \\
Marital Status & -.531 & .41 & .59 & $.167-2.075$ \\
Parenthood & -1.295 & .039 & .27 & $.080-.937$ \\
Employment & -1.709 & .001 & .18 & $.065-.505$ \\
Depression & 2.821 & .001 & 16.79 & $3.001-93.968$ \\
\hline
\end{tabular}

3 CI: Confidence interval; OR: Odds Ratio

4 Model Summary; $\left(\chi^{2}(5, N=156)=63.90, p<.001\right)$

5 